\title{
The Cost of Patient Education on a Post- Partum Unit in an Acute Care Setting
}

\author{
Audrey JOST ${ }^{\mathrm{a}, 1}, \mathrm{Kim}_{\text {VELEZ }}^{\mathrm{a}}$ and Elle FAWNS ${ }^{\mathrm{a}}$ \\ a Sisters of Charity Leavenworth Health, Broomfield, Colorado, USA
}

\begin{abstract}
Four years post-implementation, an interactive patient care system is slated for decommissioning in two hospitals. A team of experts was tasked with evaluating whether to replace or remove the functionalities of this system and the cost associated with either. The largest effects noted were the financial burden and the patient education. The cost to substitute video education with nursing time spent in patient education was determined to not be cost-effective.
\end{abstract}

Keywords. Patient education, patient education videos, postpartum, acute care

\section{Introduction}

In 2014, the second of two hospitals implemented an interactive patient care system. This system, among many other functions, allows for customized patient educational videos hosted by different vendors to be streamed via a computer connected to a television set for each patient to view in their room. Studies have shown that video patient education is very effective for patient learning [1,2]. However, the hospitals learned that the system was slated for decommissioning due to cost.

\section{Approach}

The purpose of the team of experts was to determine the effects of removing the interactive patient care system on patients, staff, and hospital operations. The largest downstream effects were immediately noted to be patient education and the financial burden of decommissioning.

\section{Method}

The team of experts in clinical, technical and hardware specialties gathered to create a crosswalk of all functionalities needing to be cost-effectively replaced or removed. This team included experts in clinical practice, clinical education, hardware, software, health informatics, leadership, hospital operations, patient experience and performance improvement. Pricing for available options were obtained or estimated based on one

\footnotetext{
${ }^{1}$ Corresponding Author, Audrey Jost, Kim Velez, Elle Fawns, 500 Eldorado Boulevard, Suite 4300, Broomfield, Colorado 80021, USA; E-mail: www.sclhealthsystem.org.
} 
hospital's post-partum unit as they were the system's top users of the video education piece of the interactive patient care system. The decommission plan document that contained all of the options and the expert recommendation was then submitted to system leadership for approval.

Twenty functionalities were identified to be evaluated and replaced or removed. Education was deemed one of the most critical functionalities needing replacement. Because postpartum demonstrated the highest use of the video education, the decommissioning team estimated replacement costs specific to that unit.

Postpartum education costs:

- On average, each education video is 2-5 minutes long. There were 6,510 hours of videos watched on the postpartum unit the previous year. If converted 1:1 in nursing time, it would take 6,510 hours per year of nursing time.

- With an average $\mathrm{RN}$ pay rate of $\$ 40 /$ hour, the cost to substitute nursing time spent in patient education was estimated to be $\$ 260,400$ annually.

The team of experts ultimately developed five options to replace or remove the functionalities of the interactive patient care system. Conservative estimates found that it would cost anywhere from $\$ 440,000$ to $\$ 1.5$ million to complete the task. None of the options effectively replaced the system currently in place or provide all of the benefits it had to offer, and all of the options exceeded the system cost of $\$ 427,000$ per year. Based on this cost-benefit analysis, the patient care system was retained.

\section{Conclusions}

The cost of education in two hospitals on just the postpartum units is staggering. When used house-wide, that number easily doubles. Discovering the cost of education increased the value of nursing time to the hospitals and made retention of this interactive patient care system a priority. Videos have been shown to increase a patient's and caregivers' knowledge and satisfaction in acute care settings and be cost effective [2,3]. This could have an enormous impact on specialized patient and caregiver success outside of an acute care setting.

\section{References}

[1] Brown T, Goldman S, Persell S et al. Development and evaluation of a patient education video promoting pneumococcal vaccination. Elsevier: Patient Education and Counseling. 100 (2017) (2016), 1024-1027.

[2] Correnti C, Chen S, Stoff B. 2017. Video based education about systemic corticosteroids enhances patient knowledge more than verbal education: A randomized controlled trial. Dermatology Online Journal. 23(9) (2017), 2-7.

[3] Denny M, Vahidy F, Vu K, Sharrief A, Savitz S. Video-based educational intervention associated with improved stroke literacy, self-efficacy, and patient satisfaction. PLoS ONE 12(3): e0171952. https://doi.org/10.1371/journal.pone.0171952 